\title{
Evaluación energética y económica de la recuperación in situ de butanol aplicando vacío cíclico en un reactor con múltiples alimentaciones
}

\author{
Víctor H. Grisales-Díaz ${ }^{1,3 *}$, Oscar A. Prado-Rubio ${ }^{1}$ y Gerard Olivar-Tost ${ }^{2,4}$ \\ (1) Fac. De Ingeniería y arquitectura, Dpto. de Ingeniería Química, Universidad Nacional de Colombia - Sede Manizales, \\ Cra. 27 No.64-60, Manizales, Colombia. (correo-e: vhgrisalesd@unal.edu.co, oaprador@unal.edu.co) \\ (2) Fac. De Ciencias Exactas y Naturales, Dpto. de Matemática y Estadística, Universidad Nacional de Colombia - Sede \\ Manizales, Cra. 27 No. 64-60, Manizales, Colombia. (correo-e: golivart@unal.edu.co) \\ (3) Fac. Ciencias de la Salud. Dpto. Microbiología. Universidad Libre, Belmonte Avenida Las Américas, Pereira, \\ Colombia (correo-e: victorh.grisalesd@unilibre.edu.co) \\ (4) Departamento de Ciencias Naturales y Tecnología, Universidad de Aysén, Coyhaique, Chile \\ (correo-e: gerard.olivar@uaysen.cl)
}

* Autor a quien debe ser dirigida la correspondencia

Recibido Ene. 2, 2020; Aceptado Mar. 4, 2020; Versión final May. 2, 2020, Publicado Ago. 2020

\begin{abstract}
Resumen
En este trabajo, se evaluó la producción de butanol por fermentación en una operación de vacío cíclica con múltiples alimentaciones de sustrato a través de una estrategia de simulación de procesos. La sacarificación del sustrato, rastrojo de maíz, fue simulada en un reactor integrado con fermentación y recuperación in situ de producto con los objetivos de alcanzar el máximo potencial económico y los mínimos requerimientos de electricidad de la bomba de calor requerida por la integración energética. Las optimizaciones multi-objetivo fueron realizadas en MATLAB ${ }^{\circledR}$. Se encontró que el vacío cíclico no permite alcanzar el máximo potencial económico y que usar una estrategia con múltiples alimentaciones es una estrategia de operación suficiente. Usando estas estrategias los requerimientos de electricidad fueron reducidos en $5 \%$ y se incrementó el potencial económico en 9\% (alrededor de 4 Millones de dólares por año) cuando se compara con un reactor integrado con una sola alimentación.
\end{abstract}

Palabras clave: vacío cíclico; múltiples alimentaciones; optimización multiobjetivo; recuperación in situ

\section{Economic and energy evaluation of in situ recovery of butanol by applying a cyclic vacuum in a multi-fed batch reactor}

\begin{abstract}
In this work, butanol production by fermentation using a cyclic vacuum and multi fed-batch operations was evaluated through a process simulation strategy. The saccharification of the substrate, corn stover, was simulated in an integrated reactor with fermentation and in situ product recovery. Optimizations were carried out to achieve as objectives the maximum economic potential and the minimum electricity requirements of the heat-pump system needed for energy integration. Multi-objective optimizations were performed in MATLAB ${ }^{\circledR}$. It was found that a cyclic vacuum was not required to achieve the maximum economic potential and that a multi-fed batch operating strategy is enough to obtain the maximum economic potential. Using these strategies, the electricity requirements were decreased by $5 \%$ and the economic potential was increased by $9 \%$ ( 4 MM USD/year) when compared to a fed-batch reactor with in situ recovery using only one feed.
\end{abstract}

Keywords: cyclic vacuum; multi fed-batch; multi-objective optimization; in situ recovery 


\section{INTRODUCCIÓN}

Debido a la actual preocupación referente a la baja calidad del aire, la seguridad energética y el cambio climático global, diferentes países alrededor del mundo han desarrollado políticas para promover el uso de las energías renovables (Hu y Chen, 2019). Ya que el sector transporte contribuye al $23 \%$ de las emisiones de efecto invernadero, es necesario reemplazar el uso de los combustibles fósiles en este sector (Hu y Chen, 2019). Entre las alternativas al combustible fósil, los combustibles a base de biomasa son una alternativa potente ya que, por ejemplo, tiene una menor emisión de gases de efecto invernadero, es renovable y es altamente disponible (Singh et al., 2019). En este sentido, tanto el bioetanol como el biobutanol son atractivos combustibles, ya que ambos son producidos a partir de la fermentación de los azúcares presentes en la biomasa (Ospino et al., 2020; Prado-Rubio et al., 2018). Entre estos dos, el butanol es el más atractivo ya que como biocombustible tiene un mejor desempeño en los automóviles que el etanol (Moon et al., 2016). Entre esas características como biocombustible podemos destacar que el butanol tiene un mayor calor de combustión, rindiendo más kilómetros por litro, y una mayor viscosidad, la cual reduce el desgaste del motor y sus pistones. Por consiguiente, el butanol fue aprobado como biocombustible en los Estados Unidos por la Agencia de Protección Ambiental como un aditivo de la gasolina con una concentración de hasta 16\%; a modo de comparación, el etanol es mezclado con la gasolina hasta 10\% (Hu y Chen, 2019).

Pese a las ventajas del butanol como biocombusible, la producción de butanol es limitada por su bajo rendimiento $(<41 \%)$ y por la baja concentración de producto $(<20 \mathrm{~g} / \mathrm{l})$ debida a su toxicidad para el microorganismo que lo produce (Chen et al., 2018). Por esta razón, una producción rentable de butanol requiere sustratos de bajo costo, tales como residuos agroindustriales (e.g. el rastrojo de maíz) y sistemas intensificados de producción (e.g. reactores de recuperación in situ de butanol) (Karimi Alavijeh y Karimi, 2019; Xue et al., 2014). En estos sistemas intensificados usando sustratos de segunda generación, se incrementa la productividad al limitarse la inhibición por producto y se disminuye el efecto del costo de sustrato, ya que el rastrojo de maíz es más económico (Karimi Alavijeh y Karimi, 2019). Adicionalmente, con estos reactores avanzados se puede reducir el consumo de agua al poder usarse mayores concentraciones de sustrato (Chen et al., 2014).

Existen diferentes alternativas de recuperación in situ, tales como la extracción líquido-líquido (Grisales-Diaz y Olivar, 2017), la pervaporación (Borisov y Volkov, 2015), el gas de arrastre (Li et al., 2016), la perstracción (Heerema et al., 2011), la adsorción (Goerlitz et al., 2018), entre otras (Outram et al., 2016). De las unidades usadas en la recuperación de producto in situ (ISPR), la evaporación a vacío es una de las más interesantes por su relativo bajo costo y simplicidad (Mariano et al., 2010). Sin embargo, esta tecnología aún tiene altos consumos energéticos. Por esta razón, en la literatura se han estudiado diferentes alternativas para la generación de vacío, los cuales incluyen sistemas de compresión con absorción y adsorción (Pereira et al., 2017, 2019), bombas de calor en múltiples etapas (Grisales-Díaz et al., 2019) y vacío cíclico (Mariano et al., 2012).

Entre las posibles alternativas, las bombas de calor han sido reportadas con los menores requerimientos energéticos por Grisales-Diaz et al., (2019). Una bomba de calor tiene bajo requerimientos energéticos porque el calor de condensación de la corriente de gases comprimidos se usa para suministrar el calor necesario para la evaporación de los solventes (Vane, 2005). Consecuentemente, los requerimientos energéticos en este sistema están solamente dados por el consumo de la electricidad en los compresores. Adicionalmente, una bomba de calor se hace más interesante en reactores a vacío, si se considera que los gases de fermentación deben ser comprimidos al menos a presión atmosférica, la cual es una presión mayor que la requerida para la condensación de los solventes a una diferencia de temperatura adecuada para la aplicación de una bomba de calor $\left(\geq 10^{\circ} \mathrm{C}\right)$. En consecuencia, en este trabajo fue estudiado un esquema de un ISPR operado a vacío y acoplado con una bomba de calor, el cual puede ser observado en la Figura 1.

Alternativamente, con el fin disminuir los requerimientos de electricidad del compresor, se ha propuesto en la literatura sistemas de operación a vacío cíclica (Mariano et al., 2012). La operación cíclica ha sido reportada por Mariano et al., (2012) como ventajosa ya que permite disminuir los requerimientos energéticos al reducir el flujo de $\mathrm{CO} 2$ en el sistema de compresión. Para alcanzar ahorros energéticos sin disminuir el potencial económico se necesitan de estrategias de simulación que permitan conocer adecuadamente los procesos y el efecto de las variables de operación en el consumo de electricidad (Cuisano et al., 2020). No obstante, no han sido reportados en la literatura análisis energéticos y de optimización usando esquemas de operación cíclica con bombas de calor en múltiples etapas de compresión. Adicionalmente, existe muy poca información tanto experimental como de simulación de este tipo de sistemas que permitan entender mejor su funcionamiento y consecuentemente no se han realizado evaluaciones tecno-económicas de este tipo de operaciones. 


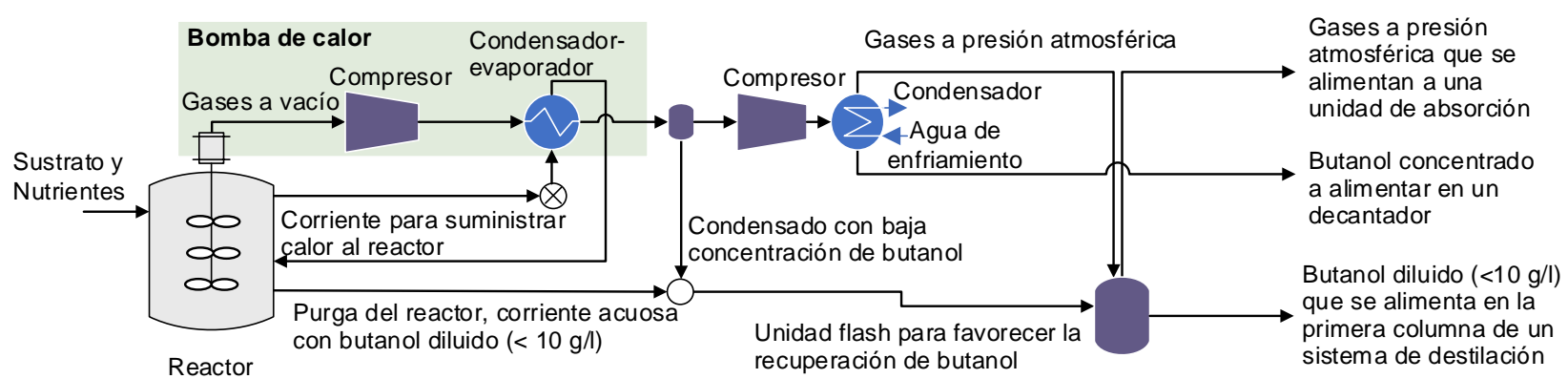

Fig. 1: Esquema de un fermentador con recuperación in situ de butanol a vacío acoplado con una bomba de calor con dos etapas de compresión

En este sentido, las estrategias de modelamiento que conlleven a la optimización de reactores discontinuos son importantes porque al disminuir el porcentaje de operación a vacío, se incrementan también los costos inversión de los compresores e intercambiadores de calor asociados a la bomba de calor (Grisales-Díaz et al., 2019). Es decir, existe un balance óptimo entre los costos de capital y los operativos. Por otro lado, los requerimientos energéticos no necesariamente serán más bajos ya que, al tener lapsos operacionales sin vacío, se debe aumentar la intensidad de la recuperación en los ciclos a vacío para alcanzar la misma velocidad de remoción del producto que un proceso de vacío continuo. Por lo tanto, para definir si aplicar vacío cíclico es mejor que continuo no se tiene una solución obvia y son necesarias estrategias de optimización para hacer una comparación cuantitativa. Es decir, se necesitan múltiples comparaciones a las mejores condiciones de operación de cada tecnología.

En aras de mejorar esfuerzos previos de la literatura, en este trabajo se realizaron optimaciones multiobjetivo de los requerimientos energéticos y el potencial económico del proceso de fermentación a vacío con operación cíclica. Adicionalmente, fueron consideradas múltiples alimentaciones en el modelo del reactor ya que variaciones en la velocidad de alimentación de sustrato pueden incrementar la productividad del reactor, es decir disminuir el volumen de reacción requerido para alcanzar un flujo de producción dado (Darmayanti et al., 2018). Incrementar la productividad en procesos ISPR es importante porque se ha encontrado que, usando unidades, tales como gas arrastre, los requerimientos energéticos dependen de la productividad alcanzada (Lodi et al., 2018). En otras palabras, múltiples alimentaciones permitirán incrementar la productividad del reactor y disminuir el porcentaje de remoción de butanol del reactor en la corriente a vacío, reduciendo así los requerimientos energéticos.

\section{METODOLOGÍA}

Los modelos matemáticos que serán implementados para llevar a cabo una optimización económica y energética de un reactor en operación discontinua fueron reportados previamente por Grisales-Diaz et al., (2019). Allí, el modelo matemático discontinuo de un reactor ISPR incluye un sistema de hidrólisis enzimática y fermentación simultánea, el cual usa el rastrojo de maíz pretratado como la fuente de sustrato. Una hidrólisis enzimática es necesaria para transformar la celulosa del rastrojo de maíz a glucosa. El microorganismo usado fue Clostridium saccharoperbutylacetonicum N1-4 (Oshiro et al., 2009; Shinto et al., 2007). Este microorganismo fue usado porque puede consumir tanto la glucosa como la xilosa presente en la fermentación después del pretratamiento e hidrólisis enzimática. En este trabajo, un sistema de hidrólisis y fermentación simultánea fue propuesto ya que esto disminuye la inhibición que genera la glucosa y xilosa en la sacarificación (Shadbahr et al., 2017).

\section{Diseño del reactor}

El modelo cinético de la hidrólisis de la celulosa fue reportado por Kadam et al., (2004). Mientras, el modelo cinético de la fermentación es un modelo metabólico riguroso que considera la producción de acetona, etanol y ácidos orgánicos y que ha sido evaluado con diferentes concentraciones de sustrato y en sistemas en lote, continuos con y sin recirculación de biomasa (Grisales-Diaz y Willis, 2018). Es importante mencionar que en el trabajo previo desarrollado por Grisales-Díaz et al., (2019) no se considera la operación cíclica y tampoco se estudiaron múltiples alimentaciones secuenciales a diferentes velocidades de dilución, métodos que son objeto de este trabajo para mejorar el desempeño tanto económico como energético de este proceso de fermentación. Por lo cual, en este trabajo fueron realizadas modificaciones, al modelo base previamente desarrollado por Grisales-Díaz et al., (2019), para incluir estas estrategias de operación. Para ello, primero se debe tener en cuenta que, en el modelo anterior, la presión en el reactor es estimada rigurosamente a partir de un modelo de equilibrio líquido-gas en el reactor y el flujo de gas $\left(F_{G}\right)$ es manipulado con un controlador proporcional de presión, tal como es descrito en la Ecuación 1: 
$F_{G}=K \cdot\left(P_{\text {set }}-P\right)$

Consecuentemente, para lograr un vacío cíclico se debe cambiar el set-point de presión $\mathrm{P}_{\text {set. }}$ desde una presión atmosférica a una presión a vacío $\left(\mathrm{P}_{v}\right)$ a unos intervalos de tiempo establecidos y viceversa. La presión de vacío en el reactor $(P)$ es estimada usando la ecuación de estado Peng-Robinson para los solventes en la fase gaseosa, con constantes de Henry para los compuestos gaseosos y en la fase líquida las actividades fueron estimadas usando UNIQUAC. Estos modelos termodinámicos y sus parámetros son descritos por Grisales-Díaz et al., (2019). En este trabajo, se asume que los intervalos de duración de la operación a vacío $\left(\Delta \mathrm{t}_{b}\right)$ pueden ser diferentes a las de operación atmosférica $\left(\Delta t_{a}\right)$ en condiciones óptimas. Adicionalmente, se consideró que el tiempo inicial de vacío ( $\left.t_{0}\right)$ puede ser mayor que cero. El tiempo máximo de fermentación $\left(t_{f}\right)$ se calcula a partir de $t_{0}$ y en relación con el tiempo de operación a vacío cíclico $\Delta \mathrm{t}$

$t_{f}=t_{o}+\Delta t$

En las simulaciones, el set-point de presión $\left(\mathrm{P}_{\text {set }}\right)$ dependen del tiempo $t_{i}$. Para cada tiempo $t_{i}$ en el cual hay un cambio de presión, $\mathrm{P}_{\text {set }}$ puede ser formulado con las siguientes ecuaciones:

$$
\left\{\begin{array}{r}
t>t_{i} y P_{\text {set }}=1 \text { bar } \rightarrow P_{\text {set }}=P_{v} y t_{i+1}=t_{i}+\Delta t_{a} \\
t>t_{i} y P_{\text {set }}=P_{v} \rightarrow P_{\text {set }}=1 \text { bary } y t_{i+1}=t_{i}+\Delta t_{b} \\
t_{i+1}+\Delta t_{a}>t_{f} \rightarrow \Delta t_{a}=t_{f}-t_{i} \\
t_{i+1}+\Delta t_{b}+\Delta t_{a}>t_{f} \rightarrow \Delta t_{b}=t_{f}-\Delta t_{a}-t_{i}
\end{array}\right\}
$$

Un ejemplo del cambio de presión en una operación cíclica con cuatro etapas es representado en la Figura 2. Es importante tener en cuenta que cuando $\mathrm{t}_{\mathrm{i}}$ es mayor que el tiempo de fermentación, los $\Delta t_{a}$ y $\Delta \mathrm{t}_{b}$ deben ser ajustados de tal forma que ti al final del ciclo sea igual a t. tal como es descrito en la ecuación 3 y se observa gráficamente en la Figura 2.

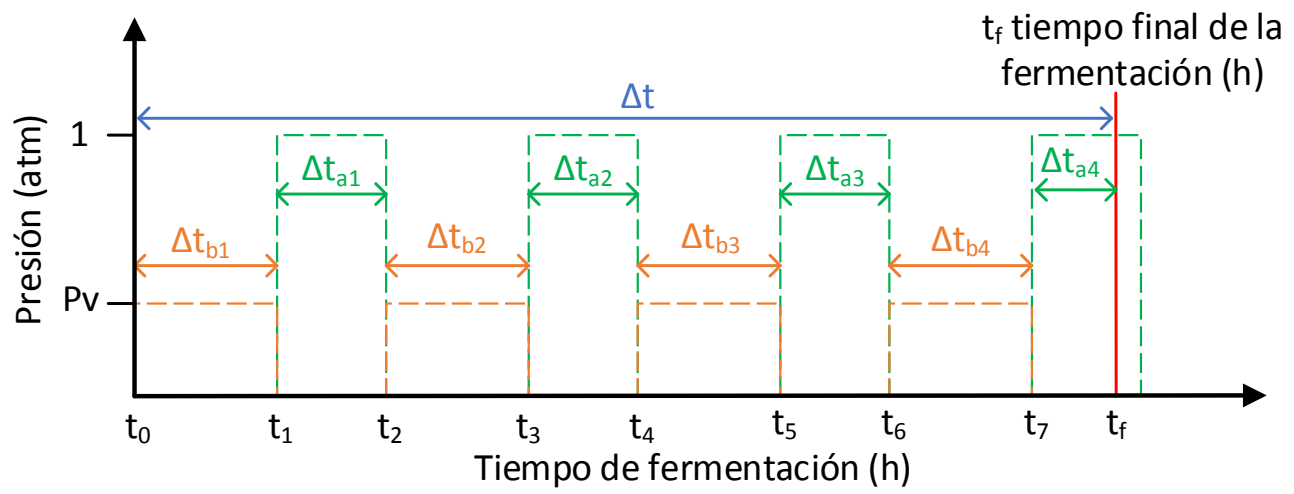

Fig. 2: Ejemplo de los perfiles de una operación cíclica con cuatro ciclos de vacío

Una vez determinados los tiempos y asumiendo un tiempo promedio de limpieza/arranque/encendido de los reactores de $4 \mathrm{~h}$, la relación del tiempo total de operación a vacío (tv) para cada fermentación es:

$t v=\frac{\sum \Delta t_{b}}{t f+4}$

Esta relación de operación a vacío es importante porque operar el reactor aplicando vacío de manera discontinua incrementa el dimensionamiento de la bomba de calor (compresores e intercambiadores de calor) y por consiguiente su inversión de capital en relación con un vacío en continuo. En el trabajo desarrollado por Grisales-Díaz et al., (2019), el cual es la base de este trabajo, fue determinado el efecto de la organización de los reactores en este dimensionamiento para un solo ciclo de vacío. No obstante, este método reportado previamente puede ser usado en este trabajo para una operación cíclica porque este incremento es estimado como una función del número de reactores y del porcentaje de tiempo a vacío.

Teniendo en cuenta que las alimentaciones múltiples no han sido consideradas anteriormente, en este trabajo se incluye una nueva modificación, la cual es descrita a continuación. En las alimentaciones múltiples, un cambio máximo de 4 flujos de alimento $\left(F_{A}\right)$ fueron estudiados. Los tiempos de duración fueron considerados usando intervalos $\Delta t_{i, i=1 \ldots 4}$, por lo cual $F_{A}$ a un determinado tiempo de operación está dado por: 


$$
\left\{\begin{array}{c}
t<\Delta t_{1} \rightarrow F_{A}=0 \\
t>\Delta t_{1} y t<\Delta t_{1}+\Delta t_{2} \rightarrow F_{A}=F_{1} \\
t>\Delta t_{1}+\Delta t_{2} y t<\Delta t_{1}+\Delta t_{2}+\Delta t_{3} \rightarrow F_{A}=F_{2} \\
t>\Delta t_{1}+\Delta t_{2}+\Delta t_{3} y t<\Delta t_{1}+\Delta t_{2}+\Delta t_{3}+\Delta t_{4} \rightarrow F_{A}=F_{3} \\
\sum_{i=1}^{a} \Delta t_{i}>t_{f} \rightarrow \Delta t_{a}=t_{f}-\sum_{i=1}^{a-1} \Delta t_{i} y \text { si } \Delta t_{a}<0 \rightarrow \Delta t_{a}=0 \forall a=1 \ldots 4
\end{array}\right\}
$$

\section{Diseño de la bomba de calor}

Una vez estimado el desempeño del reactor y los flujos de salida de estos, se estima el desempeño de la bomba de calor, la cual está compuesta de intercambiadores de calor y de compresores (ver Figura 1). El diseño de los intercambiadores de calor depende de la temperatura a la cual es enfriada la corriente gaseosa. El primer intercambiador es un evaporador-condensador el cual es usado para suministrar calor a la reacción, la cual es necesitada para que la temperatura de la fermentación no disminuya debido a la evaporación. La variable de diseño de este intercambiador de calor es fijada a una diferencia de temperatura mínima de $10^{\circ} \mathrm{C}$, es decir las corrientes calientes de salida del intercambiador son a $40{ }^{\circ} \mathrm{C}$, i.e. la temperatura de fermentación es $30^{\circ} \mathrm{C}$. Solo el primer intercambiador de calor es un evaporador/condensador. Por lo cual, los demás intercambiadores de calor son enfriados con agua de enfriamiento hasta una temperatura de $25^{\circ} \mathrm{C}$.

En lo referente a los compresores, las variables de diseño dependen de trabajo aplicado a cada uno de estos. Los trabajos requeridos por cada uno de los compresores son hallados por el método de optimización, ya que la relación de compresión óptima puede variar con el flujo gaseoso, la presión de vacío del reactor o la composición de este flujo gaseoso a vacío. Es importante mencionar que los grados de libertad del último compresor son cero ya que la presión de salida de los gases es fijada a una atmosfera. Por consiguiente, el número de variables de optimización de los compresores es igual al número de etapas de compresión menos 1. En este trabajo, las etapas de compresión son fijadas a tres, ya que este es el número óptimo, en consecuencia, se deben optimizar los trabajos requeridos por los dos primeros compresores.

\section{Evaluación económica}

En los costos operativos (OPEX) se consideraron el costo anualizado del sustrato, de las enzimas, de la electricidad y del agua de enfriamiento. Mientras en los costos de capital anualizados (CAPEX) se consideraron los costos de inversión relacionados al reactor y de una operación discontinua en la inversión de la bomba de calor. Las principales suposiciones usadas en este trabajo de las composiciones sustrato y variables económicas son reportadas en la Tabla 1. Para facilitar la comparación, estas condiciones fueron las mismas usadas previamente por Grisales-Díaz et al., (2019). En este trabajo, se consideraron tres productos, butanol, etanol y acetona, y los costos de venta de cada uno de los productos están reportados en la Tabla 1. El flujo de producción anual de butanol fue de 140000 toneladas por año, lo que da una capacidad de rastrojo de maíz alrededor de 2000 toneladas por día (Grisales-Diaz et al., 2019).

Tabla 1: Composición de sustrato y principales variables económicas usadas en este trabajo

\begin{tabular}{|l|l|l|}
\hline Variables & Unidades & Valor \\
\hline Composición de xilosa (solido soluble) & (peso/peso) & 0.047 \\
\hline Composición de celulosa (solido insoluble) & (peso $/ \mathrm{peso})$ & 0.061 \\
\hline Composición de glucosa (solido soluble) & (peso/peso) & 0.008 \\
\hline $\begin{array}{l}\text { Composición de otros solidos solubles y solidos } \\
\text { insolubles totales (en una relación 1:1) }\end{array}$ & (peso $/ \mathrm{peso})$ & 0.093 \\
\hline Composición total de sustrato libre de agua & (peso $/ \mathrm{peso})$ & 0.209 \\
\hline Costo de agua de enfriamiento & USD $/ \mathrm{MJ}$ & $2.3 \times 10^{-4}$ \\
\hline Costo de enzimas & USD $/ \mathrm{kg}-\mathrm{proteína}$ & 4.24 \\
\hline Costo del rastrojo de maíz en base seca & USD $/ \mathrm{kg}-$ lignocelulosa & 0.064 \\
\hline Costo de la electricidad & USD $/ \mathrm{kWh}$ & 0.1 \\
\hline Costo de venta del butanol & USD $/ \mathrm{kg}$ & 1.0 \\
\hline Costo de venta del etanol & USD $/ \mathrm{kg}$ & 0.8 \\
\hline Costo de venta de la acetona & USD $/ \mathrm{kg}$ & 0.7 \\
\hline
\end{tabular}

\section{Estrategia de optimización}

Las optimizaciones fueron realizadas en $M A T L A B^{\circledR}$ a través del método de algoritmos genéticos multiobjetivo 'gamultiobj', enfocados a la minimización de los requerimientos energéticos de un sistema 
compresión en tres etapas en la bomba de calor (Objetivo 1) y la maximización del potencial económico (Objetivo 2):

Objetivo $1=\sum_{i=1}^{3}$ (Trabajo realizado por el compresor $\left.{ }_{i}\right)$

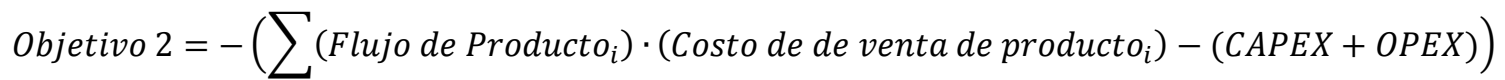

La minimización del Objetivo 2 tiene un signo negativo, ya que se desea maximizar el potencial económico. Las optimizaciones están restringidas a una ocupación del reactor menor a $80 \%$ y el contenido máximo de sólidos en el reactor debe ser menor que $110 \mathrm{~g} / \mathrm{l}$. Es importante mencionar que este tipo de optimización multiobjetivo no se ha realizado previamente para la operación vacío-discontinua de butanol. Un diagrama del procedimiento de cálculo es mostrado en la Figura 3.

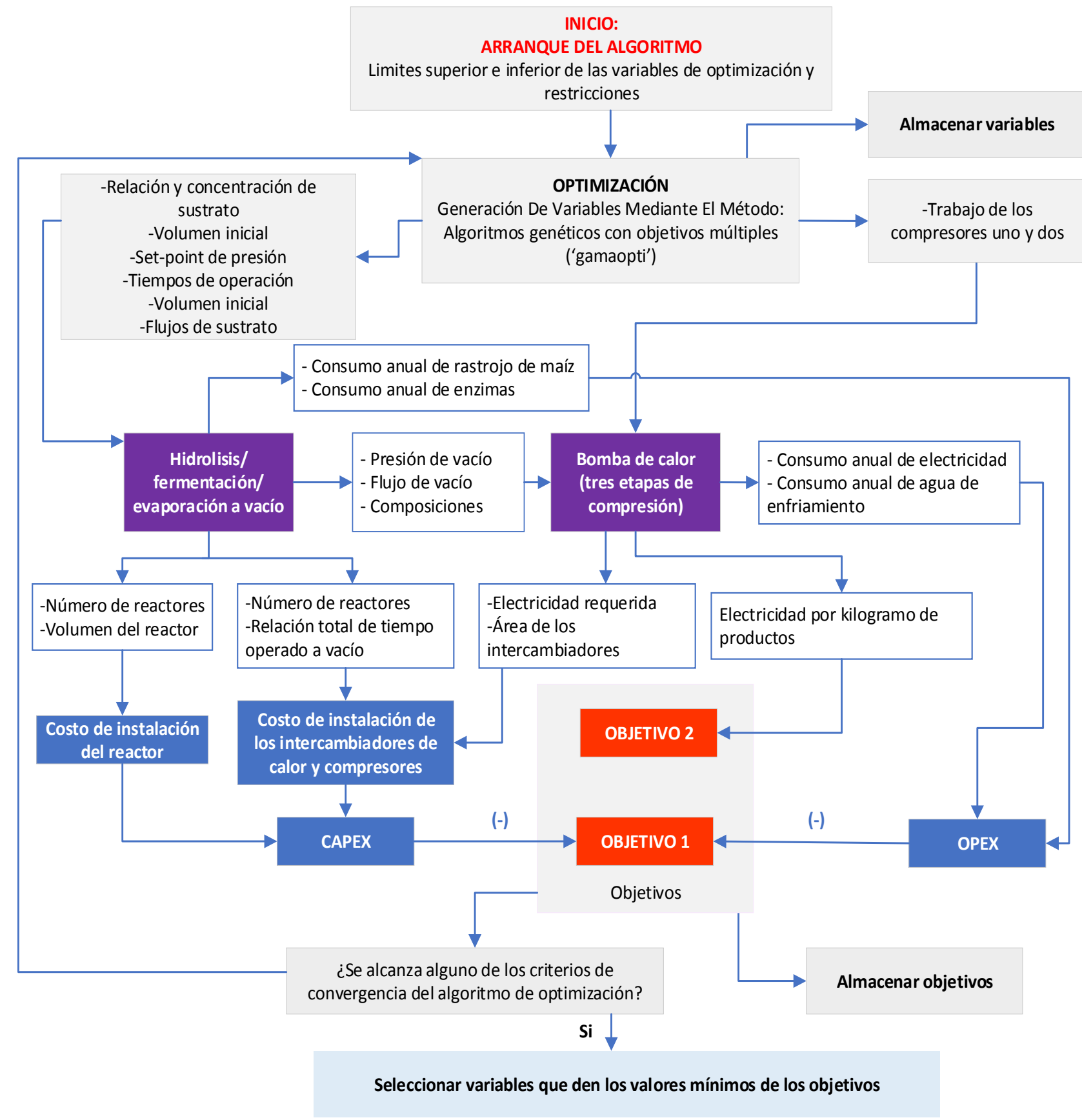

Fig. 3: Algoritmo de optimización usado en este trabajo para la producción de butanol en una operación discontinua

Con la finalidad de depurar los resultados debido a la naturaleza estocástica del método de algoritmos genéticos, las optimizaciones fueron realizadas 10 veces. Las condiciones por defecto del método fueron 
utilizadas para las optimizaciones, donde las más representativas son el número de generaciones, las cuales son 200 por el número de variables, y la función de distancia tipo fenotipo y fracción de Pareto del $35 \%$. En la Tabla 2, se encuentran las variables a optimizar, propuestas previamente por (Grisales Díaz et al., 2019), y las adicionales que han sido propuestas en este trabajo para alcanzar una operación cíclica y con múltiples alimentaciones.

Tabla 2: Variables de optimización consideradas en este trabajo para minimizar el trabajo de compresión y maximizar el potencial económico

\begin{tabular}{|l|l|}
\hline \multicolumn{2}{|c|}{ Las variables de optimización propuestas por Grisales-Díaz et al., (2019) } \\
\hline Relación y concentración de enzimas & 2 variables \\
\hline La potencia aplicada a cada compresor, un total de tres etapas de compresión son estudiadas & 2 variables \\
\hline Volumen inicial y la presión de vacío $\left(P_{\text {set }}\right)$ & 2 variables \\
\hline El tiempo de operación a vacío cíclico $(\Delta t)$ & 1 variables \\
\hline Tiempo inicial para aplicar el vacío $\left(\mathrm{t}_{0}\right)$ & 1 variable \\
\hline Flujo de alimentación $\left(F_{1}\right)$ & 1 variable \\
\hline \multicolumn{2}{|c|}{ Variables de optimización Incluidas en este trabajo con múltiples alimentaciones } \\
\hline Variables de tiempo relacionadas a la alimentación $\left(\Delta t_{1}, \Delta t_{2}, \Delta t_{3}, \Delta t_{4}\right)$ & 4 variables \\
\hline Flujos $\left(F_{2}, F_{3}\right.$ y $\left.F_{4}\right)$ & 3 variables \\
\hline Variables de optimización Incluidas en este trabajo para alcanzar una operación cíclica & \\
\hline Tiempos relacionados a los ciclos de vacío $\left(\Delta t_{a}, \Delta t_{b}\right)$ & 2 variables \\
\hline Total (grados de libertad) & 18 variables \\
\hline
\end{tabular}

Todas las variables de optimización son continuas. Los límites de búsqueda para las variables de optimización propuestas por Grisales-Díaz et al., (2019) de la Tabla 2 fueron las óptimas alcanzadas en ese trabajo previo divididas o multiplicadas por dos. Mientras que, para las nuevas variables de optimización, se consideraron los siguientes limites: las variables de tiempo fueron limitadas entre máximo 100 horas y mínimo 1 hora y los flujos fueron limitados a velocidades de dilución de máximo $0.1 \mathrm{~h}^{-1}$ y mínimo $0 \mathrm{~h}^{-1}$. En aras de comparar la necesidad de una operación cíclica y determinar el efecto de las alimentaciones múltiples en el desempeño, se realizaron las optimizaciones también sin incluir las 2 variables adiciones para alcanzar los vacíos cíclicos, descritos en la ecuación $2\left(\Delta t_{a}\right.$ y $\left.\Delta t_{b}\right)$.

\section{RESULTADOS Y DISCUSIÓN}

La producción de butanol por fermentación es limitada por su toxicidad. Por lo cual, reactores ISPR han sido sugeridos en la literatura como la mejor opción para su producción, porque en estos reactores se alcanzan relativamente bajos requerimientos energéticos con altas productividades o bajos tiempos de residencia y altos rendimientos. A manera de ejemplo, en trabajo previo, se ha encontrado que la productividad en condiciones óptimas es de $0.37 \mathrm{~g} / / \mathrm{h}$ cuando se usan reactores convencionales y material lignocelulósico pretratado como sustrato (Grisales Díaz y Olivar Tost, 2016). En comparación, en este trabajó se encontró que el rendimiento máximo usando reactores ISPR fue de $0.41 \mathrm{~g} / \mathrm{g}$, mientras la productividad máxima fue $0.85 \mathrm{~g} / / \mathrm{h}$. Es decir, se alcanzaron rendimientos cercanos al estequiométrico y se logró elevar la productividad alrededor de 2.2 veces cuando se compara con operaciones convencionales del reactor.

En la Figura 4 (a) y (b) se observa que un incremento de productividad y rendimiento es alcanzado con requerimientos energéticos superiores sin afectar negativamente el potencial económico. Adicionalmente, hay que mencionar la dispersión, es decir, que el efecto tiene diferente medida dependiendo de la solución óptima alcanzada. En las simulaciones se encontró que, para alcanzar un máximo desempeño económico se necesitan alcanzar los rendimientos más elevados posibles $(0.35-0.41 \mathrm{~g} / \mathrm{g})$, lo cual se puede ver en la Figura 4 (b). Mientras en el caso de la productividad se necesitan productividades inferiores a la máxima, alrededor de $0.5-0.7 \mathrm{~g} / \mathrm{l} / \mathrm{h}$ (Figura 4 (a)). A diferencia del rendimiento y la productividad, las enzimas no muestran una relación creciente evidente con el aumento de los requerimientos energéticos, lo que se ve reflejado en la Figura 4 (c) con una gran dispersión. Esto es causado porque las condiciones de operación del reactor pueden ser ajustadas, a los cambios de las relaciones enzimáticas, variando los tiempos de residencia en el reactor. Las concentraciones de enzimas evaluadas en las optimizaciones fueron entre 15 y $35 \mathrm{mg}$ de enzima por $g$ de celulosa y se encontraron los máximos potenciales económicos con concentraciones de enzima entre 18 y $25 \mathrm{mg} / \mathrm{g}$.

Los requerimientos energéticos dependen de dos factores, tanto del flujo de remoción $\left(F_{G}\right)$ como de las relaciones de compresión en la bomba de calor. El primero es influyente porque se aumenta el flujo de alimentación a los compresores, mientras el segundo es relevante ya que relaciones de compresión óptimas permiten disminuir el flujo del gas debido a la condensación y también reduce el aumento de la temperatura 
en la corriente gaseosa, la cual es causada por la compresión de esta. Si se utiliza diseños ineficientes de la bomba de calor, en algunos casos no se alcanzan productividades elevadas incluso cuando se consiguen altos requerimientos energéticos de compresión, tal como se ve reflejado en la Figura 4 (a). Esto evidencia la necesidad de optimizar la bomba de calor en conjunto con las condiciones de operación del reactor.
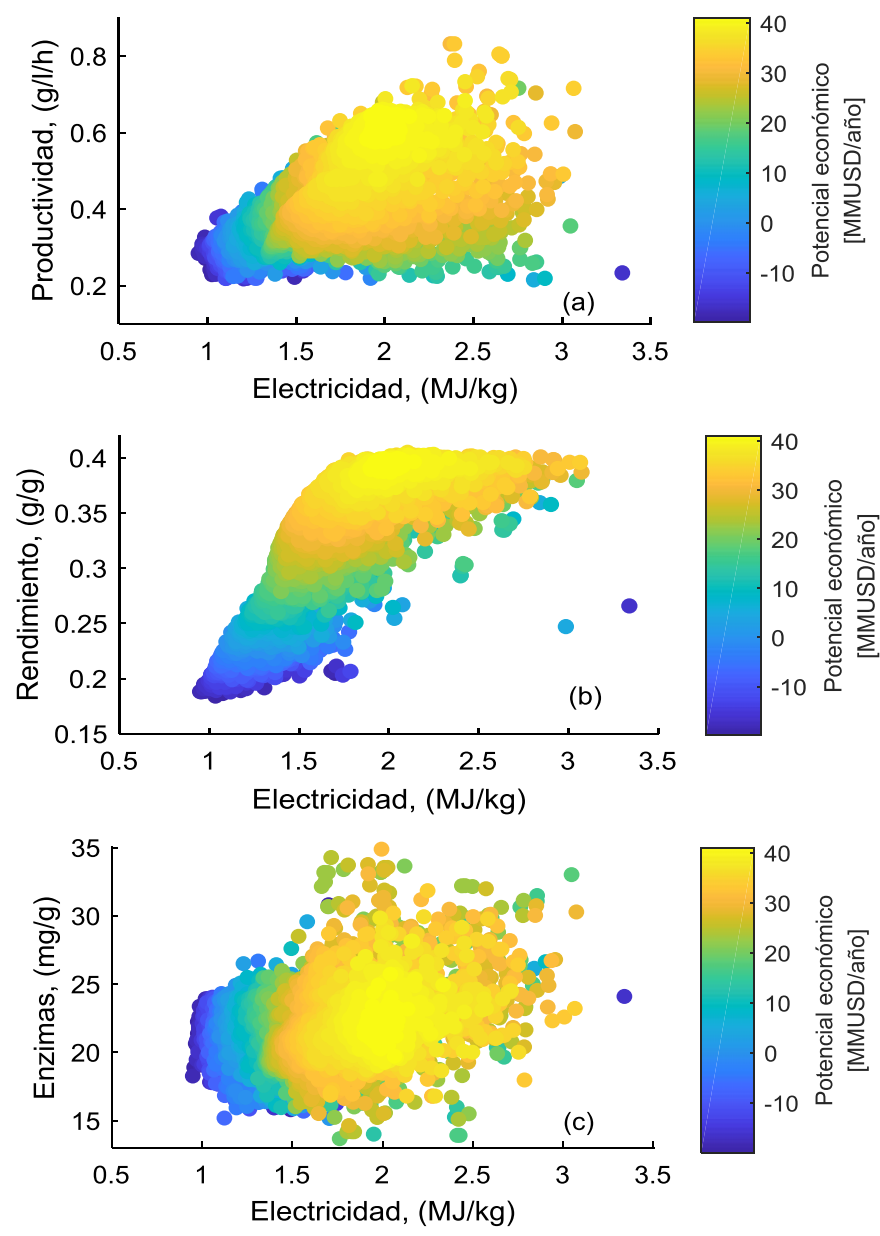

Fig. 4: Indicadores de desempeño de un fermentador con recuperación in situ de butanol a vacío cíclico acoplado con una bomba de calor. a) productividad; b) rendimiento; c) enzimas

En la simulación, no se alcanzó en condiciones óptimas a cumplir un ciclo entre vacío y presión atmosférica ya que $\Delta t_{a}$ más $\Delta t_{b}$ fue igual que el tiempo de fermentación. En la Tabla 3 se encuentran las variables de operación óptimas encontradas en este trabajo. En las simulaciones fue encontrado que un vacío cíclico no es necesario para obtener el máximo potencial económico. Sin embargo, se encontró que aplicar una presión atmosférica antes de terminar la fermentación (después de 90 h) es necesario para alcanzar menores requerimientos energéticos a condiciones de máximo potencial económico. Teniendo en cuenta lo anterior, en los perfiles de la concentración de butanol cuando se deja de aplicar vacío al reactor se incrementa la concentración de butanol en el reactor de 6 a $8 \mathrm{~g} / \mathrm{l}$ (Figura 5). Este incremento de la concentración de butanol dentro del reactor es interesante, porque además puede ser beneficioso para la recuperación subsecuente de butanol por destilación.

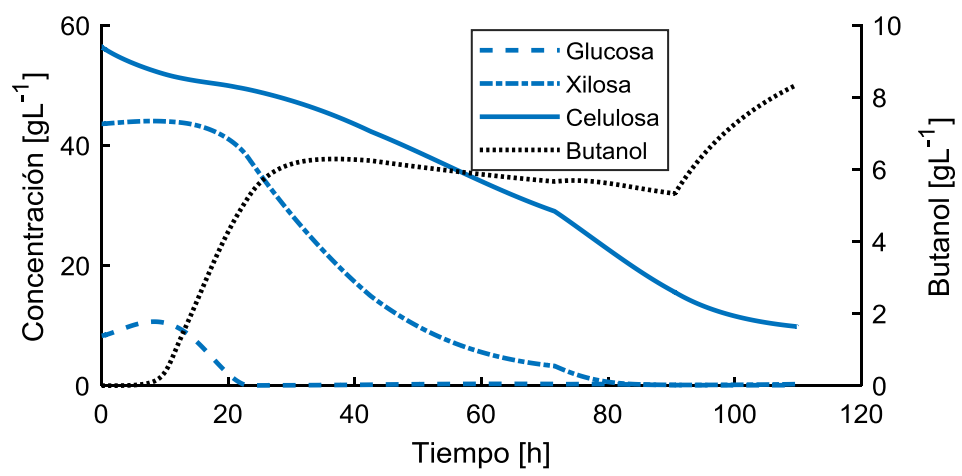

Fig. 5. Perfiles de concentración usando condiciones de operación óptima 
Tabla 3: Condiciones óptimas de operación del reactor

\begin{tabular}{|c|c|c|c|}
\hline $\begin{array}{l}\text { Concentración de enzima endo- } \beta \text {-1,4-glucanasa/exo- } \beta \text { - } \\
\text { 1,4-cellobiohidrolasa }(\mathrm{CBH}),(\mathrm{mg} / \mathrm{g})\end{array}$ & 3.7 & Flujo de alimentación, $F_{1}\left(\mathrm{~h}^{-1}\right)$ & 0.0043 \\
\hline Concentración de enzima $\beta$-glucosidasa (mg/g) & 17.0 & Flujo de alimentación, $F_{2}\left(\mathrm{~h}^{-1}\right)$ & 0.0053 \\
\hline Presión de set-point $P_{\text {se }}$ (bar) & 0.0507 & Flujo de alimentación, $F_{3}\left(\mathrm{~h}^{-1}\right)$ & 0.0067 \\
\hline Volumen inicial/Volumen del reactor $\left(\mathrm{m}^{3}\right)$ & 2770 & Flujo de alimentación, $F_{4}\left(\mathrm{~h}^{-1}\right)$ & 0.0016 \\
\hline Presión 1 en el tren de compresión (bar) & 0.182 & Duración alimentación, $\Delta t_{1}(\mathrm{~h})$ & 1.0 \\
\hline Presión 2 en el tren de compresión (bar) & 0.356 & Duración alimentación, $\Delta t_{2}(\mathrm{~h})$ & 21.23 \\
\hline Tiempo final de fermentación & 109.8 & Duración alimentación, $\Delta t_{3}(\mathrm{~h})$ & 21.16 \\
\hline Cambió a vacío $\Delta t_{o}(\mathrm{~h})$ & 0.7 & Duración alimentación, $\Delta t_{4}$ (h) & 29.00 \\
\hline Cambió a presión atmosférica $\Delta t_{a}(\mathrm{~h})$ & 90.5 & Cambió a presión atmosférica $\Delta t_{b}(\mathrm{~h})$ & 18.7 \\
\hline
\end{tabular}

En relación con las múltiples alimentaciones, el flujo de alimentación es constantemente incrementado desde 0.0043 a $0.0067 \mathrm{~h}^{-1}$ porque una mayor concentración de biomasa es alcanzada en el reactor (resultados no mostrados) y después de 50 horas de fermentación es reducido a $0.0016 \mathrm{~h}^{-1}$ para alcanzar mayores conversiones de sustrato al final de la fermentación. Las alimentaciones al reactor fueron detenidas después de $\sim 72 \mathrm{~h}$ de fermentación. Teniendo en cuenta que un vacío cíclico no es una estrategia que permita alcanzar el máximo potencial económico, se realizó una optimización multiobjetivo con todas las variables excepto las variables de operación cíclica.

En la Figura 6 pueden ser observados los resultados de estas optimizaciones. Cuando las optimizaciones fueron realizadas incluyendo solo las variables de múltiples alimentaciones, aunque se alcanza el máximo potencial económico, se alcanzó con un requerimiento de electricidad $5 \%$ más elevado. En relación a lo reportado previamente por Grisales-Díaz et al., (2019), en un el sistema de operación a vacío con más bajos requerimientos energéticos, el potencial económico y los requerimientos energéticos alcanzados, en este trabajo, fueron $9 \%$ más altos y bajos, respectivamente; obteniendo un potencial económico de 41.2 millones de dólares por año y unos requerimientos energéticos de compresión de $2 \mathrm{MJ} / \mathrm{kg} A B E$.

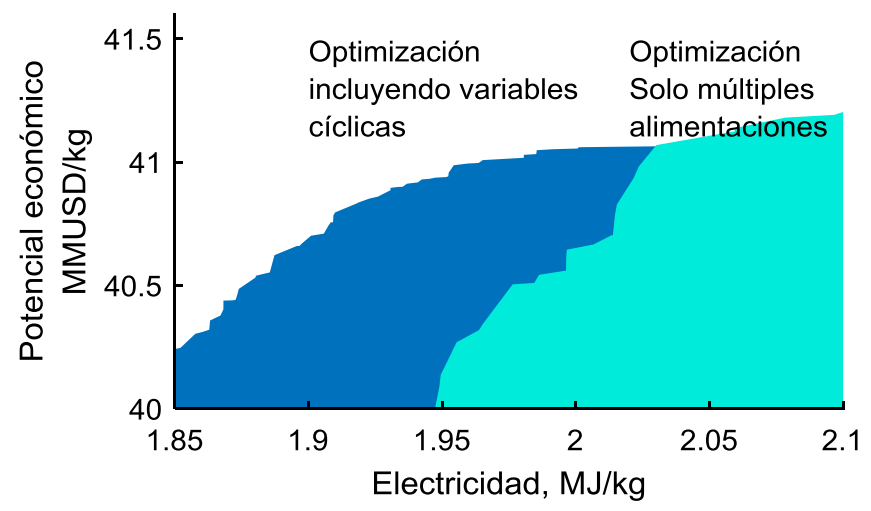

Fig. 6: Efecto del vacío cíclico en el potencial económico y requerimientos energéticos en comparación con un reactor operado con vacío continuo

Los resultados de los requerimientos energéticos encontrados en este trabajo aplicando vacío cíclico son contradictorios a los previamente reportados por el único trabajo que ha evaluado este tipo de operación, ya que Mariano et al., (2012) reporto ahorros energéticos de 39\% cuando un vacío cíclico es aplicado. Sin embargo, las condiciones de operación que se estudiaron por Mariano et al., (2012) no fueron óptimas, lo cual es importante tal como se encontró en este trabajo, ya que reducciones en los requerimientos energéticos pueden ser alcanzados con reducciones en la productividad o el rendimiento. Finalmente, Mariano et al., (2012) realizo la comparación usando las mismas presiones para la operación cíclica y no cíclica, lo cual no considera la capacidad de mejorar los requerimientos energéticos de la operación en continúo incrementando la presión de operación.

\section{CONCLUSIONES}

Los resultados de optimización mostraron que usar una estrategia de operación con múltiples alimentaciones es suficiente para alcanzar el máximo potencial económico. Aplicar ciclos a unas condiciones de operación dadas, reduce los requerimientos energéticos, no obstante, también reduce el 
potencial económico al incrementar los costos de capital de la bomba de calor y al disminuir la productividad del reactor. Al no reducir los requerimientos energéticos para alcanzar el potencial económico más elevado, los diagramas de Pareto obtenidos demuestran que los ciclos de vacío no son atractivos como una alternativa al vacío en continuo. Sin embargo, se encontró que no es necesario aplicar vacío faltando $19 \mathrm{~h}$ para terminar la fermentación y se puede operar en este periodo a condiciones atmosféricas, lo cual reduce en un $5 \%$ los requerimientos energéticos a condiciones de máximo potencial económico.

\section{AGRADECIMIENTOS}

Los autores agradecen a Colciencias pues este trabajo fue financiado gracias a la convocatoria de estancia postdoctoral 811 de COLCIENCIAS, Colombia 2018. Adicionalmente, agradecemos a Colciencias por soportar la publicación de este artículo a través del proyecto "Modelado y Simulación del Metabolismo Urbano de Bogotá D.C. Código $111974558276 "$.

\section{REFERENCIAS}

Borisov, I.L. y Volkov, V.V., Thermopervaporation Concept for Biobutanol Recovery: The Effect of Process Parameters, https://doi.org/10.1016/j.seppur.2015.03.023, Sep. Purif. Technol., 146, 33-41 (2015)

Chen, C., Cai, D. y otros cuatro autores, Bio-Plasticizer Production by Hybrid Acetone-Butanol-Ethanol Fermentation with Full Cell Catalysis of Candida sp. 99-125, https://doi.org/10.1016/j.biortech.2018.02.066, Bioresour. Technol., 257, 217-222 (2018)

Chen, Y., Ren, H., y otros trece autores, Enhancement of n-Butanol Production by in situ Butanol Removal using Permeating-Heating-Gas Stripping in Acetone-Butanol-Ethanol Fermentation, https://doi.org/10.1016/j.biortech.2014.04.107, Bioresour. Technol., 164, 276-284 (2014)

Cuisano, J.C., Chirinos, L.R. y Barrantes, E.J., Eficiencia Energética en Sistemas Eléctricos de Micro, Pequeñas y Medianas Empresas del Sector de Alimentos. Simulación para Optimizar Costos de Consumo de Energía Eléctrica, https://doi.org/10.4067/S0718-07642020000200267, Inf. Tecnol., 31, 267-276 (2020)

Darmayanti, R.F., Tashiro, Y. y otros cuatro autores, Novel Biobutanol Fermentation at a Large Extractant Volume Ratio Using Immobilized Clostridium saccharoperbutylacetonicum N1-4, https://doi.org/10.1016/j.jbiosc.2018.06.006, J. Biosci. Bioeng., 126, 750-757 (2018)

Goerlitz, R., Weisleder, y otros cinco autores, Bio-butanol Downstream Processing: Regeneration of Adsorbents and Selective Exclusion of Fermentation by-products, https://doi.org/10.1007/s10450-017-9918-x, Adsorption, 24, 95-104 (2018)

Grisales Díaz, V.H., Olivar Tost, G., Butanol Production from Lignocellulose by Simultaneous Fermentation, Saccharification, and Pervaporation or Vacuum Evaporation, https://doi.org/10.1016/j.biortech.2016.06.091, Bioresour. Technol., 218, 174-182 (2016)

Grisales Díaz, V.H. y Olivar Tost, G., Techno-economic Analysis of Extraction-based Separation Systems for Acetone, Butanol, and Ethanol Recovery and Purification, https://doi.org/10.1186/s40643-017-0142-z, Bioresour. Bioprocess., 4, 12 (2017)

Grisales Díaz, V.H., von Stosch, M. y Willis, M.J., Butanol Production Via Vacuum Fermentation: An Economic Evaluation of Operating Strategies, https://doi.org/10.1016/j.ces.2018.10.016, Chem. Eng. Sci., 195, 707-719 (2019)

Grisales Díaz, V.H. y Willis, M.J., Kinetic Modelling and Simulation of Batch, Continuous and Cell-recycling Fermentations for Acetone-butanol-ethanol Production Using Clostridium saccharoperbutylacetonicum N1-4, https://doi.org/10.1016/j.bej.2018.05.011, Biochem. Eng. J., 137, 30-39 (2018)

Heerema, L., Roelands, M. y otros tres autores, J., In-situ Product Removal from Fermentations by Membrane Extraction: Conceptual Process Design and Economics, https://doi.org/10.1021/ie102551g, Ind. Eng. Chem. Res., 50, 9197-9208 (2011)

Hu, K. y Chen, Y., Equilibrium Fuel Supply and Carbon Credit Pricing Under Market Competition and Environmental Regulations: A California Case Study, https://doi.org/10.1016/j.apenergy.2018.12.041, Appl. Energy, 236, 815-824 (2019)

Kadam, K.L., Rydholm, E.C. y McMillan, J.D., Development and Validation of a Kinetic Model for Enzymatic Saccharification of Lignocellulosic Biomass, https://doi.org/10.1021/bp034316x, Biotechnol. Prog., 20, 698-705 (2004)

Karimi Alavijeh, M. y Karimi, K., Biobutanol Production from Corn Stover in the US, https://doi.org/10.1016/j.indcrop.2018.12.054, Ind. Crops Prod., 129, 641-653 (2019)

Li, S.-Y., Chiang, C.-J., Tseng, I.-T., He, C.-R. y Chao, Y.-P., Bioreactors and In Situ Product Recovery Techniques for Acetone-butanol-ethanol Fermentation, https://doi.org/10.1093/femsle/fnw107, FEMS Microbiol. Lett., 363, fnw107 (2016)

Lodi, G., De Guido, G. y Pellegrini, L.A. Simulation and Energy Analysis of the ABE Fermentation Integrated with Gas Stripping, https://doi.org/10.1016/j.biombioe.2018.06.012, Biomass and Bioenergy, 116, 227-235 (2018)

Mariano, A.P., Costa, C.B.B., y otros cinco autores, Optimisation of a Fermentation Process for Butanol Production by Particle swarm optimisation (PSO), https://doi.org/10.1002/jctb.2383, J. Chem. Technol. Biotechnol., 85, 934-949 (2010)

Mariano, A.P., Filho, R.M. y Ezeji, T.C., Energy Requirements During Butanol Production and in situ Recovery by Cyclic Vacuum, https://doi.org/10.1016/j.renene.2012.04.041, Renew. Energy, 47, 183-187 (2012) 
Moon, H.G., Jang, Y.S. y otros cuatro autores, One Hundred Years of Clostridial Butanol Fermentation, https://doi.org/10.1093/femsle/fnw001, FEMS Microbiol. Lett. 363 (2016)

Oshiro, M., Shinto, H. y otros cinco autores, Kinetic Modeling and Sensitivity Analysis of Xylose Metabolism in Lactococcus lactis IO-1, https://doi.org/10.1016/j.jbiosc.2009.05.003, J. Biosci. Bioeng., 108, 376-384 (2009)

Ospino, K., Gómez, E. y Rios, L., Evaluación de Técnicas de Pretratamiento en Buchón de Agua (Eichhornia crassipes) para la Producción de Bioetanol, https://doi.org/10.4067/S0718-07642020000100215, Inf. Tecnol., 31, 215-226 (2020)

Outram, V., Lalander, C.-A. y otros tres autores, A comparison of the energy use of in situ product recovery techniques for the acetone butanol ethanol fermentation, https://doi.org/10.1016/j.biortech.2016.09.002, Bioresour. Technol., 220, 590-600 (2016)

Pereira, J.P.C., Lopez-Gomez, G. y otros tres autores, Prospects and Challenges for the Recovery of 2-Butanol Produced by Vacuum Fermentation - a Techno-Economic Analysis, https://doi.org/10.1002/biot.201600657, Biotechnol. J., 12, 1600657 (2017)

Pereira, J.P.C., Overbeek, W. y otros cinco autores, Integrated Vacuum Stripping and Adsorption for the Efficient Recovery of (Biobased) 2-Butanol, https://doi.org/10.1021/acs.iecr.8b03043, Ind. Eng. Chem. Res., 58, 296-305 (2019)

Prado-Rubio, O.A., Rodriguez-Gomez, D. y Morales-Rodriguez, R., Model-Based Approach to Enhance Configurations for 2G Butanol Production, Recent Innov. Chem. Eng. (Formerly Recent Patents Chem. Eng.),11, 99-111 (2018)

Shadbahr, J., Khan, F. y Zhang, Y., Kinetic Modeling and Dynamic Analysis of Simultaneous Saccharification and Fermentation of Cellulose to Bioethanol, https://doi.org/10.1016/j.enconman.2016.08.025, Energy Convers. Manag., 141, 236-243 (2017)

Shinto, H., Tashiro, Y. y otros siete autores, Kinetic Modeling and Sensitivity Analysis of Acetone-Butanol-Ethanol Production, https://doi.org/10.1016/j.jbiotec.2007.05.005, J. Biotechnol., 131, 45-56 (2007)

Singh, V., Singh, H. y Das, D., Optimization of the Medium Composition for the Improvement of Hydrogen and Butanol Production using Clostridium Saccharoperbutylacetonicum DSM 14923, https://doi.org/10.1016/j.jhydene.2019.08.125, Int. J. Hydrogen Energy, 44, 26905-26919 (2019)

Vane, L.M., A Review of Pervaporation for Product Recovery from Biomass Fermentation Processes, https://doi.org/10.1002/jctb.1265, J. Chem. Technol. Biotechnol., 80, 603-629 (2005)

Xue, C., Zhao, J.B. y otros cuatro autores, Integrated Butanol Recovery for an Advanced Biofuel: Current State and Prospects, https://doi.org/10.1007/s00253-014-5561-6, Appl. Microbiol. Biotechnol., 98, 3463-3474 (2014) 
